\title{
FRACTIONAL OSTROWSKI TYPE INEQUALITIES FOR FUNCTIONS OF BOUNDED VARIATON WITH TWO VARIABLES
}

\author{
S. ERDEN, H. BUDAK, AND M. Z. SARIKAYA
}

Received 15 October, 2019

\begin{abstract}
We first establish some fractional equalities for functions of bounded variation with two variables. Then we derive some fractional Ostrowski and Trapezoid type inequalities for functions of bounded variation with two variables. In addition, we give some Midpoint inequalities as special cases of our main results.
\end{abstract}

2010 Mathematics Subject Classification: 26B30; 26D07; 26D10

Keywords: fuctions of bounded variation, fractional integrals, Ostrowski type inequalities, midpoint type inequalities

\section{INTRODUCTION}

The study of various types of integral inequalities has been the focus of great attention for well over a century by a number of mathematicians, interested both in pure and applied mathematics. One of the many fundamental mathematical discoveries of A. M. Ostrowski [22] is the following classical integral inequality associated with the differentiable mappings:

Theorem 1. Let $f:[a, b] \rightarrow \mathbb{R}$ be a differentiable mapping on $(a, b)$ whose derivative $f^{\prime}:(a, b) \rightarrow \mathbb{R}$ is bounded on $(a, b)$, i.e. $\left\|f^{\prime}\right\|_{\infty}:=\sup _{t \in(a, b)}\left|f^{\prime}(t)\right|<\infty$. Then, we have the inequality

$$
\left|f(x)-\frac{1}{b-a} \int_{a}^{b} f(t) d t\right| \leq\left[\frac{1}{4}+\frac{\left(x-\frac{a+b}{2}\right)^{2}}{(b-a)^{2}}\right](b-a)\left\|f^{\prime}\right\|_{\infty},
$$

for all $x \in[a, b]$. The constant $\frac{1}{4}$ is the best possible.

Ostrowski inequality has applications in quadrature, probability and optimization theory, stochastic, statistics, information and integral operator theory. Until now, a large number of research papers and books have been written on Ostrowski inequalities and their numerous applications. In general, the generalizations to the Ostrowski 
type inequalities are obtained by introducing arbitrary parameters in the Peano kernels involved. The parameters can be so adjusted to recapture the previous results as well as to obtain some new estimates of such inequalities.

On the other hand, the inequalities discovered by C. Hermite and J. Hadamard for convex functions are considered significant in the literature (see, e.g.,[12], [23, p.137]). These inequalities state that if $f: I \rightarrow \mathbb{R}$ is a convex function on the interval $I$ of real numbers and $a, b \in I$ with $a<b$, then

$$
f\left(\frac{a+b}{2}\right) \leq \frac{1}{b-a} \int_{a}^{b} f(x) d x \leq \frac{f(a)+f(b)}{2} .
$$

Both inequalities hold in the reversed direction if $f$ is concave. We note that Hermite-Hadamard inequality may be regarded as a refinement of the concept of convexity and it follows easily from Jensen's inequality. Over the years, many studies have focused on to establish generalization of the inequality (1.1) and to obtain new bounds for left-hand side (Midpoint) and right-hand side (Trapezoid) of the inequality (1.1).

The remainder of this work is organized as follows: In Section 2, we present the definitions of fractional integrals and functions of bounded variation. We also mention some inequalities related fractional integrals. In Section 3, we establish three identities for double fractional integrals. Finally, some new fractional Ostrowski and Trapezoid type integral inequalities are proved for functions of bounded variation with two variables in Section 4. We also give some fractional Midpoint type inequalities in Section 4.

\section{PRELIMINARIES}

Firstly, we give the definitions of Riemann-Liouville fractional integrals:

Definition 1. Let $f \in L_{1}[a, b]$. The Riemann-Liouville integrals $J_{a+}^{\alpha} f$ and $J_{b-}^{\alpha} f$ of order $\alpha>0$ with $a \geq 0$ are defined by

$$
J_{a+}^{\alpha} f(x)=\frac{1}{\Gamma(\alpha)} \int_{a}^{x}(x-t)^{\alpha-1} f(t) d t, x>a
$$

and

$$
J_{b-}^{\alpha} f(x)=\frac{1}{\Gamma(\alpha)} \int_{x}^{b}(t-x)^{\alpha-1} f(t) d t, x<b
$$

respectively. Here, $\Gamma(\alpha)$ is the Gamma function and $J_{a+}^{0} f(x)=J_{b-}^{0} f(x)=f(x)$.

For more information and properties about fractional integrals, please refer to ([18], [21], [24]).

It is remarkable that Sarikaya et al.[30] firstly give the interesting integral inequalities of Hermite-Hadamard type involving Riemann-Liouville fractional integrals. Whereupon several papers focus on fractional Hermite-Hadamard and Ostrowski type inequalities, for example ([1], [10], [19], [27], [29], [28]-[26], [31]). 
Now, we give the definitions Riemann-Liouville fractional integrals of two variable functions:

Definition 2 ([25]). Let $f \in L_{1}([a, b] \times[c, d])$. The Riemann-Liouville fractional integrals $J_{a+, c+}^{\alpha, \beta}, J_{a+, d-}^{\alpha, \beta}, J_{b-, c+}^{\alpha, \beta}$ and $J_{b-, d-}^{\alpha, \beta}$ are defined by

$$
\begin{aligned}
& J_{a+, c+}^{\alpha, \beta} f(x, y)=\frac{1}{\Gamma(\alpha) \Gamma(\beta)} \int_{a}^{x} \int_{c}^{y}(x-t)^{\alpha-1}(y-s)^{\beta-1} f(t, s) d s d t, \quad x>a, y>c, \\
& J_{a+, d-}^{\alpha, \beta} f(x, y)=\frac{1}{\Gamma(\alpha) \Gamma(\beta)} \int_{a}^{x} \int_{y}^{d}(x-t)^{\alpha-1}(s-y)^{\beta-1} f(t, s) d s d t, \quad x>a, y<d, \\
& J_{b-, c+}^{\alpha, \beta} f(x, y)=\frac{1}{\Gamma(\alpha) \Gamma(\beta)} \int_{x}^{b} \int_{c}^{y}(t-x)^{\alpha-1}(y-s)^{\beta-1} f(t, s) d s d t, \quad x<b, y>c,
\end{aligned}
$$

and

$$
J_{b-, d-}^{\alpha, \beta} f(x, y)=\frac{1}{\Gamma(\alpha) \Gamma(\beta)} \int_{x}^{b} \int_{y}^{d}(t-x)^{\alpha-1}(s-y)^{\beta-1} f(t, s) d s d t, \quad x<b, y<d .
$$

Hermite-Hadamard inequality and Ostrowski inequality for fractional integrals of two-variable functions are obtained in [25] and [20], respectively. There are several papers on fractional Hermite-Hadamard and fractional Ostrowski type inequalities for two-variable functions, you can find some of them in the references.

Functions of bounded variation of one variable are of great interest and usefulness because of their valuable properties, such as particularly with respect to additivity, decomposability into monotone functions, continuity, differentiability, measurability, integrability, and so on, have been much studied. There are many of papers on inequalities for functions of bounded variation of one variable, some of them please see ([2], [6], [7], [8], [11], [13],[16], [17]). Moreover Dragomir obtained some fractional inequalities involving functions of bounded variation ([14], [15])

Functions of bounded variation with two variables are defined as follows:

Definition 3 ([9] ). Assume that $f(x, y)$ is defined over the rectangle $Q=[a, b] \times$ $[c, d]$. Let $P$ be a partition of $Q$ with

$$
P: a=x_{0}<x_{1}<\ldots<x_{n}=b, \text { and } c=y_{0}<y_{1}<\ldots<y_{m}=d ;
$$

and for all $i, j$ let

$$
\Delta_{11} g\left(x_{i}, y_{j}\right)=g\left(x_{i-1}, y_{j-1}\right)-g\left(x_{i-1}, y_{j}\right)-g\left(x_{i}, y_{j-1}\right)+g\left(x_{i}, y_{j}\right) .
$$

The function $f(x, y)$ is said to be of bounded variation if the sum

$$
\sum_{i=0}^{n-1} \sum_{j=0}^{m-1}\left|\Delta_{11} f\left(x_{i}, y_{j}\right)\right|
$$


is bounded for all nets.

Therefore, one can define the concept of total variation of a function of variables, as follows:

Let $f$ be of bounded variation on $Q=[a, b] \times[c, d]$, and let $\sum(P)$ denote the sum $\sum_{i=1}^{n} \sum_{j=1}^{m}\left|\Delta_{11} f\left(x_{i}, y_{j}\right)\right|$ corresponding to the partition $P$ of $Q$. The number

$$
\bigvee_{Q}(f):=\bigvee_{c}^{d} \bigvee_{a}^{b}(f):=\sup \left\{\sum(P): P \in P(Q)\right\},
$$

is called the total variation of $f$ on $Q$.

There are also some paper on inequalities for functions of bounded variation with two variables ([3], [4], [5]). However, there is a few papers fractional integral inequalities for functions of bounded variation with two variables. The aim of this paper is to establish some fractional Ostrowski, Midpoint, and Trapezoid type inequalities for functions of bounded variation with two variables.

\section{SOME IDENTITIES FOR DOUBLE INTEGRALS}

It is obtained three double integral identities involving Riemann-Liouville fractional integrals in this section. These equalities are the main material of inequalities developed throughout the article.

Lemma 1. Let $f: \Lambda:=[a, b] \times[c, d] \rightarrow \mathbb{R}$ be an absolutely continuous function on $\Lambda$ in $\mathbb{R}^{2}$. Then, for any $(x, y) \in[a, b] \times[c, d]$, we have

$$
\begin{aligned}
& \frac{1}{\Gamma(\alpha) \Gamma(\beta)} \int_{a}^{b} \int_{c}^{d} Q(x, t, y, s) F(x, t, y, s) d s d t \\
& =J_{a+, c+}^{\alpha, \beta} f(x, y)+J_{a+, d-}^{\alpha, \beta} f(x, y)+J_{b-, c+}^{\alpha, \beta} f(x, y)+J_{b-, d-}^{\alpha, \beta} f(x, y) \\
& \quad-\frac{(y-c)^{\beta}+(d-y)^{\beta}}{\Gamma(\beta+1)}\left[J_{a+}^{\alpha} f(x, y)+J_{b-}^{\alpha} f(x, y)\right] \\
& \quad-\frac{(x-a)^{\alpha}+(b-x)^{\alpha}}{\Gamma(\alpha+1)}\left[J_{c+,}^{\beta} f(x, y)+J_{d-, f}^{\beta} f(x, y)\right] \\
& \quad+\frac{\left[(b-x)^{\alpha}+(x-a)^{\alpha}\right]\left[(d-y)^{\beta}+(y-c)^{\beta}\right]}{\Gamma(\alpha+1) \Gamma(\beta+1)} f(x, y)
\end{aligned}
$$

where $F(x, t, y, s)$ and $Q(x, t, y, s)$ are defined by

$$
F(x, t, y, s):=f(t, s)-f(t, y)-f(x, s)+f(x, y)
$$

and

$$
Q(x, t, y, s)
$$




$$
:=\left\{\begin{array}{cc}
(x-t)^{\alpha-1}(y-s)^{\beta-1}, & a \leq t<x \text { and } c \leq s<y \\
(x-t)^{\alpha-1}(s-y)^{\beta-1}, & a \leq t<x \text { and } y \leq s \leq d \\
(t-x)^{\alpha-1}(y-s)^{\beta-1}, & x \leq t \leq b \text { and } c \leq s<y \\
(t-x)^{\alpha-1}(s-y)^{\beta-1}, & x \leq t \leq b \text { and } y \leq s \leq d,
\end{array}\right.
$$

respectively.

Proof. From the definition of $Q(x, t, y, s)$, we write the equality

$$
\begin{aligned}
& \frac{1}{\Gamma(\alpha) \Gamma(\beta)} \int_{a}^{b} \int_{c}^{d} Q(x, t, y, s) F(x, t, y, s) d s d t \\
& =\frac{1}{\Gamma(\alpha) \Gamma(\beta)} \int_{a}^{x} \int_{c}^{y}(x-t)^{\alpha-1}(y-s)^{\beta-1} F(x, t, y, s) d s d t \\
& +\frac{1}{\Gamma(\alpha) \Gamma(\beta)} \int_{a}^{x} \int_{y}^{d}(x-t)^{\alpha-1}(s-y)^{\beta-1} F(x, t, y, s) d s d t \\
& +\frac{1}{\Gamma(\alpha) \Gamma(\beta)} \int_{x}^{b} \int_{c}^{y}(t-x)^{\alpha-1}(y-s)^{\beta-1} F(x, t, y, s) d s d t \\
& +\frac{1}{\Gamma(\alpha) \Gamma(\beta)} \int_{x}^{b} \int_{y}^{d}(t-x)^{\alpha-1}(s-y)^{\beta-1} F(x, t, y, s) d s d t .
\end{aligned}
$$

Using the elementary analysis operations for the first integral in the right hand of the above identity, due to the definition of $F(x, t, y, s)$, we get the equality

$$
\begin{aligned}
& \frac{1}{\Gamma(\alpha) \Gamma(\beta)} \int_{a}^{x} \int_{c}^{y}(x-t)^{\alpha-1}(y-s)^{\beta-1}[f(t, s)-f(t, y)-f(x, s)+f(x, y)] d s d t \\
& =J_{a+, c+}^{\alpha, \beta} f(x, y)-\frac{(y-c)^{\beta}}{\Gamma(\beta+1)} J_{a+,}^{\alpha} f(x, y) \\
& \quad-\frac{(x-a)^{\alpha}}{\Gamma(\alpha+1)} J_{c+,}^{\beta} f(x, y)+\frac{(x-a)^{\alpha}(y-c)^{\beta}}{\Gamma(\alpha+1) \Gamma(\beta+1)} f(x, y) .
\end{aligned}
$$

If we combine the resulting integrals after having been similarly analysed the other integrals, then we reach the desired equality (3.1). 
Lemma 2. Let $f: \Lambda:=[a, b] \times[c, d] \rightarrow \mathbb{R}$ be an absolutely continuous function on $\Lambda$ in $\mathbb{R}^{2}$. Then, for any $(x, y) \in[a, b] \times[c, d]$, we have

$$
\begin{aligned}
& \frac{1}{\Gamma(\alpha) \Gamma(\beta)} \int_{a}^{b} \int_{c}^{d} Q(t, s) F(x, t, y, s) d s d t \\
& =J_{x+, y+}^{\alpha, \beta} f(b, d)+J_{x+, y-}^{\alpha, \beta} f(b, c)+J_{x-, y+}^{\alpha, \beta} f(a, d)+J_{x-, y-}^{\alpha, \beta} f(a, c) \\
& \quad-\frac{(d-y)^{\beta}+(y-c)^{\beta}}{\Gamma(\beta+1)}\left[J_{x+}^{\alpha} f(b, y)+J_{x-}^{\alpha} f(a, y)\right] \\
& \quad-\frac{(b-x)^{\alpha}+(x-a)^{\alpha}}{\Gamma(\alpha+1)}\left[J_{y+,}^{\beta} f(x, d)+J_{y-,}^{\beta} f(x, c)\right] \\
& \quad+\frac{\left[(b-x)^{\alpha}+(x-a)^{\alpha}\right]\left[(d-y)^{\beta}+(y-c)^{\beta}\right]}{\Gamma(\alpha+1) \Gamma(\beta+1)} f(x, y)
\end{aligned}
$$

where $F(x, t, y, s)$ is defined as in (3.2) and $Q(t, s)$ is defined by

$$
\begin{aligned}
& Q(t, s) \\
& := \begin{cases}(t-a)^{\alpha-1}(s-c)^{\beta-1}, & a \leq t<x \text { and } c \leq s<y \\
(t-a)^{\alpha-1}(d-s)^{\beta-1}, & a \leq t<x \text { and } y \leq s \leq d \\
(b-t)^{\alpha-1}(s-c)^{\beta-1}, & x \leq t \leq b \text { and } c \leq s<y \\
(b-t)^{\alpha-1}(d-s)^{\beta-1}, & x \leq t \leq b \text { and } y \leq s \leq d .\end{cases}
\end{aligned}
$$

Proof. By the definition of $Q(t, s)$, we find that

$$
\begin{aligned}
& \frac{1}{\Gamma(\alpha) \Gamma(\beta)} \int_{a}^{b} \int_{c}^{d} Q(t, s) F(x, t, y, s) d s d t \\
= & \frac{1}{\Gamma(\alpha) \Gamma(\beta)} \int_{x}^{b} \int_{y}^{d}(b-t)^{\alpha-1}(d-s)^{\beta-1} F(x, t, y, s) d s d t \\
& +\frac{1}{\Gamma(\alpha) \Gamma(\beta)} \int_{x}^{b} \int_{c}^{y}(b-t)^{\alpha-1}(s-c)^{\beta-1} F(x, t, y, s) d s d t \\
& +\frac{1}{\Gamma(\alpha) \Gamma(\beta)} \int_{a}^{x} \int_{y}^{d}(t-a)^{\alpha-1}(d-s)^{\beta-1} F(x, t, y, s) d s d t \\
& +\frac{1}{\Gamma(\alpha) \Gamma(\beta)} \int_{a}^{x} \int_{c}^{y}(t-a)^{\alpha-1}(s-c)^{\beta-1} F(x, t, y, s) d s d t .
\end{aligned}
$$


Using the elementary analysis rules for the first integral in the right hand of the above equality, owing to the definition of $F(x, t, y, s)$, we obtain

$$
\begin{aligned}
& \frac{1}{\Gamma(\alpha) \Gamma(\beta)} \int_{x}^{b} \int_{y}^{d}(b-t)^{\alpha-1}(d-s)^{\beta-1}[f(t, s)-f(t, y)-f(x, s)+f(x, y)] d s d t \\
& =J_{x+, y+}^{\alpha, \beta} f(b, d)-\frac{(d-y)^{\beta}}{\Gamma(\beta+1)} J_{x+}^{\alpha} f(b, y) \\
& \quad-\frac{(b-x)^{\alpha}}{\Gamma(\alpha+1)} J_{y+,}^{\beta} f(x, d)+\frac{(b-x)^{\alpha}(d-y)^{\beta}}{\Gamma(\alpha+1) \Gamma(\beta+1)} f(x, y) .
\end{aligned}
$$

Should we add the finding statements side by side after having been similarly examined the other integrals, we can easily deduce the required identity (3.4).

Lemma 3. Let $f: \Lambda:=[a, b] \times[c, d] \rightarrow \mathbb{R}$ be an absolutely continuous function on $\Lambda$ in $\mathbb{R}^{2}$. Then, for any $(x, y) \in[a, b] \times[c, d]$, we have

$$
\begin{aligned}
\frac{1}{4} & \frac{1}{\Gamma(\alpha) \Gamma(\beta)}\left\{\int _ { a } ^ { b } \int _ { c } ^ { d } \left[(t-a)^{\alpha-1}(s-c)^{\beta-1}+(t-a)^{\alpha-1}(d-s)^{\beta-1}\right.\right. \\
& \left.\left.+(b-t)^{\alpha-1}(s-c)^{\beta-1}+(b-t)^{\alpha-1}(d-s)^{\beta-1}\right] F(x, t, y, s) d s d t\right\} \\
= & \frac{J_{b-, d-}^{\alpha, \beta} f(a, c)+J_{b-, c+}^{\alpha, \beta} f(a, d)+J_{a+, d-}^{\alpha, \beta} f(b, c)+J_{a+, c+}^{\alpha, \beta} f(b, d)}{4} \\
& -\frac{1}{2} \frac{(d-c)^{\beta}}{\Gamma(\beta+1)}\left[J_{b-}^{\alpha} f(a, y)+J_{a+}^{\alpha} f(b, y)\right] \\
& -\frac{1}{2} \frac{(b-a)^{\alpha}}{\Gamma(\alpha+1)}\left[J_{d-,}^{\beta} f(x, c)+J_{c+,}^{\beta} f(x, d)\right]+\frac{(b-a)^{\alpha}(d-c)^{\beta}}{\Gamma(\alpha+1) \Gamma(\beta+1)} f(x, y)
\end{aligned}
$$

where $F(x, t, y, s)$ is defined as in (3.2).

Proof. By the definition of $F(x, t, y, s)$ and simple calculations, for the first expression, we find that

$$
\begin{aligned}
& \frac{1}{\Gamma(\alpha) \Gamma(\beta)} \int_{a}^{b} \int_{c}^{d}(t-a)^{\alpha-1}(y-c)^{\beta-1}[f(t, s)-f(t, y)-f(x, s)+f(x, y)] d s d t \\
& =J_{b-, d-}^{\alpha, \beta} f(a, c)-\frac{(d-c)^{\beta}}{\Gamma(\beta+1)} J_{b-}^{\alpha} f(a, y) \\
& \quad-\frac{(b-a)^{\alpha}}{\Gamma(\alpha+1)} J_{d-,}^{\beta} f(x, c)+\frac{(b-a)^{\alpha}(d-c)^{\beta}}{\Gamma(\alpha+1) \Gamma(\beta+1)} f(x, y) .
\end{aligned}
$$


If we similarly observe the other expressions and later we add all these identities side by side, then the desired equality can be obtained.

Corollary 1. Under the assumptions of Lemma 1 with $x=\frac{a+b}{2}$ and $y=\frac{c+d}{2}$, then the following Midpoint type equality holds:

$$
\begin{aligned}
& \frac{1}{\Gamma(\alpha) \Gamma(\beta)} \int_{a}^{b} \int_{c}^{d} Q\left(\frac{a+b}{2}, t, \frac{c+d}{2}, s\right) F\left(\frac{a+b}{2}, t, \frac{c+d}{2}, s\right) s d t \\
& =J_{a+, c+}^{\alpha, \beta} f\left(\frac{a+b}{2}, \frac{c+d}{2}\right)+J_{a+, d-}^{\alpha, \beta} f\left(\frac{a+b}{2}, \frac{c+d}{2}\right) \\
& \quad+J_{b-,, c+}^{\alpha, \beta} f\left(\frac{a+b}{2}, \frac{c+d}{2}\right)+J_{b-, d-}^{\alpha, \beta} f\left(\frac{a+b}{2}, \frac{c+d}{2}\right) \\
& -\frac{(d-c)^{\beta}}{2^{\beta-1} \Gamma(\beta+1)}\left[J_{a+}^{\alpha} f\left(\frac{a+b}{2}, \frac{c+d}{2}\right)+J_{b-}^{\alpha} f\left(\frac{a+b}{2}, \frac{c+d}{2}\right)\right] \\
& -\frac{(b-a)^{\alpha}}{2^{\alpha-1} \Gamma(\alpha+1)}\left[J_{c+, f}^{\beta} f\left(\frac{a+b}{2}, \frac{c+d}{2}\right)+J_{d-,}^{\beta} f\left(\frac{a+b}{2}, \frac{c+d}{2}\right)\right] \\
& +\frac{(b-a)^{\alpha}(d-c)^{\beta}}{2^{\alpha+\beta-2} \Gamma(\alpha+1) \Gamma(\beta+1)}\left(\frac{a+b}{2}, \frac{c+d}{2}\right)
\end{aligned}
$$

where $F\left(\frac{a+b}{2}, t, \frac{c+d}{2}, s\right)$ and $Q\left(\frac{a+b}{2}, t, \frac{c+d}{2}, s\right)$ are defined as in (3.2) and (3.3), respectively.

Corollary 2. With the assumption of Lemma 2, we have the Midpoint type identity

$$
\begin{aligned}
& \frac{1}{\Gamma(\alpha) \Gamma(\beta)} \int_{a}^{b} \int_{c}^{d} Q(t, s) F\left(\frac{a+b}{2}, t, \frac{c+d}{2}, s\right) d s d t \\
& =J_{\frac{a+b}{2}+, \frac{c+d}{2}+}^{\alpha, \beta} f(b, d)+J_{\frac{a+b}{2}+, \frac{c+d}{2}-}^{\alpha, \beta} f(b, c)+J_{\frac{a+b}{2}-, \frac{c+d}{2}+}^{\alpha, \beta} f(a, d)+J_{\frac{a+b}{2}-, \frac{c+d}{2}-}^{\alpha, \beta} f(a, c) \\
& \quad-\frac{(d-c)^{\beta}}{2^{\beta-1} \Gamma(\beta+1)}\left[J_{\frac{a+b}{2}+}^{\alpha} f\left(b, \frac{c+d}{2}\right)+J_{\frac{a+b}{2}-}^{\alpha} f\left(a, \frac{c+d}{2}\right)\right] \\
& \quad-\frac{(b-a)^{\alpha}}{2^{\alpha-1} \Gamma(\alpha+1)}\left[J_{\frac{c+d}{2}+,}^{\beta} f\left(\frac{a+b}{2}, d\right)+J_{\frac{c+d}{2}-,}^{\beta} f\left(\frac{a+b}{2}, c\right)\right] \\
& +\frac{(b-a)^{\alpha}(d-c)^{\beta}}{2^{\alpha+\beta-2} \Gamma(\alpha+1) \Gamma(\beta+1)} f\left(\frac{a+b}{2}, \frac{c+d}{2}\right)
\end{aligned}
$$

where $F\left(\frac{a+b}{2}, t, \frac{c+d}{2}, s\right)$ and $Q(t, s)$ are defined as in (3.2) and (3.5), respectively. 
Corollary 3. Suppose that all the assumptions of Lemma 3 hold. If we choose $x=\frac{a+b}{2}$ and $y=\frac{c+d}{2}$, then we possess

$$
\begin{aligned}
\frac{1}{4} & \frac{1}{\Gamma(\alpha) \Gamma(\beta)}\left\{\int _ { a } ^ { b } \int _ { c } ^ { d } \left[(t-a)^{\alpha-1}(s-c)^{\beta-1}+(t-a)^{\alpha-1}(d-s)^{\beta-1}\right.\right. \\
& \left.\left.+(b-t)^{\alpha-1}(s-c)^{\beta-1}+(b-t)^{\alpha-1}(d-s)^{\beta-1}\right] F\left(\frac{a+b}{2}, t, \frac{c+d}{2}, s\right) d s d t\right\} \\
= & \frac{J_{b-, d-}^{\alpha, \beta} f(a, c)+J_{b-, c+}^{\alpha, \beta} f(a, d)+J_{a+, d-}^{\alpha, \beta} f(b, c)+J_{a+, c+-}^{\alpha, \beta} f(b, d)}{4} \\
& -\frac{1}{2} \frac{(d-c)^{\beta}}{\Gamma(\beta+1)}\left[J_{b-}^{\alpha} f\left(a, \frac{c+d}{2}\right)+J_{a+}^{\alpha} f\left(b, \frac{c+d}{2}\right)\right] \\
& -\frac{1}{2} \frac{(b-a)^{\alpha}}{\Gamma(\alpha+1)}\left[J_{d-,}^{\beta} f\left(\frac{a+b}{2}, c\right)+J_{c+,}^{\beta} f\left(\frac{a+b}{2}, d\right)\right] \\
& +\frac{(b-a)^{\alpha}(d-c)^{\beta}}{\Gamma(\alpha+1) \Gamma(\beta+1)} f\left(\frac{a+b}{2}, \frac{c+d}{2}\right)
\end{aligned}
$$

where $F\left(\frac{a+b}{2}, t, \frac{c+d}{2}, s\right)$ is defined as in (3.2).

\section{Double INTEGRAL INEQUALITIES FOR FUNCTIONS OF BOUNDED VARIATIONS}

In this section, we establish some fractional Ostrowski and trapezoid type inequalities for function of bounded variation.

Theorem 2. Let $f: \Lambda \rightarrow \mathbb{R}$ be a function of bounded variation on $\Lambda$ in $\mathbb{R}^{2}$. Then we have the inequality

$$
\begin{aligned}
& \mid J_{a+, c+}^{\alpha, \beta} f(x, y)+J_{a+, d-}^{\alpha, \beta} f(x, y)+J_{b-, c+}^{\alpha, \beta} f(x, y)+J_{b-, d-}^{\alpha, \beta} f(x, y) \\
& \quad-\frac{(y-c)^{\beta}+(d-y)^{\beta}}{\Gamma(\beta+1)}\left[J_{a+}^{\alpha} f(x, y)+J_{b-}^{\alpha} f(x, y)\right] \\
& \quad-\frac{(x-a)^{\alpha}+(b-x)^{\alpha}}{\Gamma(\alpha+1)}\left[J_{c+,}^{\beta} f(x, y)+J_{d-,}^{\beta} f(x, y)\right] \\
& \quad+\frac{\left[(b-x)^{\alpha}+(x-a)^{\alpha}\right]\left[(d-y)^{\beta}+(y-c)^{\beta}\right]}{\Gamma(\alpha+1) \Gamma(\beta+1)} f(x, y) \mid \\
& \leq \frac{(x-a)^{\alpha}(y-c)^{\beta}}{\Gamma(\alpha+1) \Gamma(\beta+1)} \bigvee_{a}^{x} \bigvee_{c}^{y}(f)+\frac{(x-a)^{\alpha}(d-y)^{\beta}}{\Gamma(\alpha+1) \Gamma(\beta+1)} \bigvee_{a}^{x} \bigvee_{y}^{d}(f)
\end{aligned}
$$




$$
\begin{aligned}
& +\frac{(b-x)^{\alpha}(y-c)^{\beta}}{\Gamma(\alpha+1) \Gamma(\beta+1)} \bigvee_{x}^{b} \bigvee_{c}^{y}(f)+\frac{(b-x)^{\alpha}(d-y)^{\beta}}{\Gamma(\alpha+1) \Gamma(\beta+1)} \bigvee_{x}^{b} \bigvee_{y}^{d}(f) \\
\leq & \frac{1}{\Gamma(\alpha+1) \Gamma(\beta+1)}\left[\frac{1}{2}(b-a)+\left|x-\frac{a+b}{2}\right|\right]^{\alpha} \\
& \times\left[\frac{1}{2}(d-c)+\left|y-\frac{c+d}{2}\right|\right]_{a}^{\beta} \bigvee_{c}^{b} \bigvee_{c}^{d}(f)
\end{aligned}
$$

for any $(x, y) \in \Lambda$.

Proof. If we take absolute value of both sides of the equality (3.1), due to the definition of $Q(x, t, y, s)$ and the well-known triangle inequality, we get

$$
\begin{aligned}
& \mid J_{a+, c+}^{\alpha, \beta} f(x, y)+J_{a+, d-}^{\alpha, \beta} f(x, y)+J_{b-, c+}^{\alpha, \beta} f(x, y)+J_{b-, d-}^{\alpha, \beta} f(x, y) \\
& \quad-\frac{(y-c)^{\beta}+(d-y)^{\beta}}{\Gamma(\beta+1)}\left[J_{a+}^{\alpha} f(x, y)+J_{b-}^{\alpha} f(x, y)\right] \\
& \quad-\frac{(x-a)^{\alpha}+(b-x)^{\alpha}}{\Gamma(\alpha+1)}\left[J_{c+,}^{\beta} f(x, y)+J_{d-,}^{\beta} f(x, y)\right] \\
& \quad+\frac{\left[(b-x)^{\alpha}+(x-a)^{\alpha}\right]\left[(d-y)^{\beta}+(y-c)^{\beta}\right]}{\Gamma(\alpha+1) \Gamma(\beta+1)} f(x, y) \mid \\
& \leq \frac{1}{\Gamma(\alpha) \Gamma(\beta)} \int_{a}^{b} \int_{c}^{d}|Q(x, t, y, s)||F(x, t, y, s)| d s d t
\end{aligned}
$$

and

$$
\begin{aligned}
& \frac{1}{\Gamma(\alpha) \Gamma(\beta)} \int_{a}^{b} \int_{c}^{d}|Q(x, t, y, s)||F(x, t, y, s)| d s d t \\
& =\frac{1}{\Gamma(\alpha) \Gamma(\beta)} \int_{a}^{x} \int_{c}^{y}(x-t)^{\alpha-1}(y-s)^{\beta-1}|F(x, t, y, s)| d s d t \\
& +\frac{1}{\Gamma(\alpha) \Gamma(\beta)} \int_{a}^{x} \int_{y}^{d}(x-t)^{\alpha-1}(s-y)^{\beta-1}|F(x, t, y, s)| d s d t \\
& +\frac{1}{\Gamma(\alpha) \Gamma(\beta)} \int_{x}^{b} \int_{c}^{y}(t-x)^{\alpha-1}(y-s)^{\beta-1}|F(x, t, y, s)| d s d t
\end{aligned}
$$




$$
+\frac{1}{\Gamma(\alpha) \Gamma(\beta)} \int_{x}^{b} \int_{y}^{d}(t-x)^{\alpha-1}(s-y)^{\beta-1}|F(x, t, y, s)| d s d t
$$

for any $(x, y) \in \Lambda$.

Seeing that $f: \Lambda \rightarrow \mathbb{R}$ is of bounded variation on $[a, x] \times[c, y]$, we get

$$
\begin{aligned}
|F(x, t, y, s)| & =|f(t, s)-f(t, y)-f(x, s)+f(x, y)| \\
& \leq \bigvee_{t}^{x} \bigvee_{s}^{y}(f) \leq \bigvee_{a}^{x} \bigvee_{c}^{y}(f)
\end{aligned}
$$

Hence, if we consider the first integral in the right hand side of the statement (4.2), then we can easily conclude that

$$
\begin{aligned}
& \frac{1}{\Gamma(\alpha) \Gamma(\beta)} \int_{a}^{x} \int_{c}^{y}(x-t)^{\alpha-1}(y-s)^{\beta-1}|F(x, t, y, s)| d s d t \\
& \leq \frac{(x-a)^{\alpha}(y-c)^{\beta}}{\Gamma(\alpha+1) \Gamma(\beta+1)} \bigvee_{a}^{x} \bigvee_{c}^{y}(f) .
\end{aligned}
$$

Should the other integrals are also observed by taking account of the fact that $f$ : $\Lambda \rightarrow \mathbb{R}$ is of bounded variation on $[a, x] \times[y, d],[x, b] \times[c, y]$ and $[x, b] \times[y, d]$, one can readily attain the first inequality in (4.1).

The second inequality is obvious from the facts that

$$
\begin{aligned}
\max \{a c, a d, b c, b d\} & =\max \{a, b\} \max \{c, d\}, \\
\max \left\{a^{n}, b^{n}\right\} & =(\max \{a, b\})^{n}=\left(\frac{a+b+|a+b|}{2}\right)^{n}
\end{aligned}
$$

for $a, b, c, d, n>0$. This completes the proof.

Corollary 4. Under the assumptions of Theorem 2 with $x=\frac{a+b}{2}$ and $y=\frac{c+d}{2}$, we have the midpoint type inequality

$$
\begin{aligned}
& \mid J_{a+, c+}^{\alpha, \beta} f\left(\frac{a+b}{2}, \frac{c+d}{2}\right)+J_{a+, d-}^{\alpha, \beta} f\left(\frac{a+b}{2}, \frac{c+d}{2}\right) \\
& \quad+J_{b-, c+}^{\alpha, \beta} f\left(\frac{a+b}{2}, \frac{c+d}{2}\right)+J_{b-, d-}^{\alpha, \beta} f\left(\frac{a+b}{2}, \frac{c+d}{2}\right) \\
& \quad-\frac{(d-c)^{\beta}}{2^{\beta-1} \Gamma(\beta+1)}\left[J_{a+}^{\alpha} f\left(\frac{a+b}{2}, \frac{c+d}{2}\right)+J_{b-}^{\alpha} f\left(\frac{a+b}{2}, \frac{c+d}{2}\right)\right] \\
& \quad-\frac{(b-a)^{\alpha}}{2^{\alpha-1} \Gamma(\alpha+1)}\left[J_{c+, f}^{\beta} f\left(\frac{a+b}{2}, \frac{c+d}{2}\right)+J_{d-,}^{\beta} f\left(\frac{a+b}{2}, \frac{c+d}{2}\right)\right]
\end{aligned}
$$


182

$$
\begin{aligned}
& +\frac{(b-a)^{\alpha}(d-c)^{\beta}}{2^{\alpha+\beta-2} \Gamma(\alpha+1) \Gamma(\beta+1)} f\left(\frac{a+b}{2}, \frac{c+d}{2}\right) \mid \\
\leq & \frac{(b-a)^{\alpha}(c-d)^{\beta}}{2^{\alpha+\beta} \Gamma(\alpha+1) \Gamma(\beta+1)} \bigvee_{a}^{b} \bigvee_{c}^{d}(f) .
\end{aligned}
$$

Theorem 3. Let $f: \Lambda \rightarrow \mathbb{R}$ be a function of bounded variation on $\Lambda$ in $\mathbb{R}^{2}$. Then, one has the following inequality

$$
\begin{aligned}
& \mid J_{x+, y+}^{\alpha, \beta} f(b, d)+J_{x+, y-}^{\alpha, \beta} f(b, c)+J_{x-, y+}^{\alpha, \beta} f(a, d)+J_{x-, y-}^{\alpha, \beta} f(a, c) \\
& \quad-\frac{(d-y)^{\beta}+(y-c)^{\beta}}{\Gamma(\beta+1)}\left[J_{x+}^{\alpha} f(b, y)+J_{x-}^{\alpha} f(a, y)\right] \\
& \quad-\frac{(b-x)^{\alpha}+(x-a)^{\alpha}}{\Gamma(\alpha+1)}\left[J_{y+,}^{\beta} f(x, d)+J_{y-,}^{\beta} f(x, c)\right] \\
& \quad+\frac{\left[(b-x)^{\alpha}+(x-a)^{\alpha}\right]\left[(d-y)^{\beta}+(y-c)^{\beta}\right]}{\Gamma(\alpha+1) \Gamma(\beta+1)} f(x, y) \mid \\
& \leq \frac{(x-a)^{\alpha}(y-c)^{\beta}}{\Gamma(\alpha+1) \Gamma(\beta+1)} \bigvee_{a}^{x} \bigvee_{c}^{y}(f)+\frac{(x-a)^{\alpha}(d-y)^{\beta}}{\Gamma(\alpha+1) \Gamma(\beta+1)} \bigvee_{a}^{x} \bigvee_{y}^{d}(f) \\
& \quad+\frac{(b-x)^{\alpha}(y-c)^{\beta}}{\Gamma(\alpha+1) \Gamma(\beta+1)} \bigvee_{x}^{b} \bigvee_{c}^{y}(f)+\frac{(b-x)^{\alpha}(d-y)^{\beta}}{\Gamma(\alpha+1) \Gamma(\beta+1)} \bigvee_{x}^{b} \bigvee_{y}^{d}(f) \\
& \leq \\
& \quad \frac{1}{\Gamma(\alpha+1) \Gamma(\beta+1)}\left[\frac{1}{2}(b-a)+\left.\right|_{x-\frac{a+b}{2}} ^{\alpha}\right]^{\alpha} \\
& \quad \times\left[\frac{1}{2}(d-c)+\left|y-\frac{c+d}{2}\right|^{\beta} \bigvee_{a}^{b} \bigvee_{c}^{d}(f)\right.
\end{aligned}
$$

for any $(x, y) \in \Lambda$.

Proof. Taking modulus of both sides of the equality (3.4), on account of the definition of $Q(t, s)$ and the triangle inequality, we find that

$$
\begin{aligned}
& \mid J_{x+, y+}^{\alpha, \beta} f(b, d)+J_{x+, y-}^{\alpha, \beta} f(b, c)+J_{x-, y+}^{\alpha, \beta} f(a, d)+J_{x-, y-}^{\alpha, \beta} f(a, c) \\
& \quad-\frac{(d-y)^{\beta}+(y-c)^{\beta}}{\Gamma(\beta+1)}\left[J_{x+}^{\alpha} f(b, y)+J_{x-}^{\alpha} f(a, y)\right] \\
& \quad-\frac{(b-x)^{\alpha}+(x-a)^{\alpha}}{\Gamma(\alpha+1)}\left[J_{y+,}^{\beta} f(x, d)+J_{y-,}^{\beta} f(x, c)\right]
\end{aligned}
$$




$$
\begin{aligned}
& +\frac{\left[(b-x)^{\alpha}+(x-a)^{\alpha}\right]\left[(d-y)^{\beta}+(y-c)^{\beta}\right]}{\Gamma(\alpha+1) \Gamma(\beta+1)} f(x, y) \mid \\
\leq & \frac{1}{\Gamma(\alpha) \Gamma(\beta)} \int_{a}^{b} \int_{c}^{d}|Q(t, s)||F(x, t, y, s)| d s d t
\end{aligned}
$$

and

$$
\begin{aligned}
& \frac{1}{\Gamma(\alpha) \Gamma(\beta)} \int_{a}^{b} \int_{c}^{d}|Q(t, s)||F(x, t, y, s)| d s d t \\
& =\frac{1}{\Gamma(\alpha) \Gamma(\beta)} \int_{x}^{b} \int_{y}^{d}(b-t)^{\alpha-1}(d-s)^{\beta-1}|F(x, t, y, s)| d s d t \\
& +\frac{1}{\Gamma(\alpha) \Gamma(\beta)} \int_{x}^{b} \int_{c}^{y}(b-t)^{\alpha-1}(s-c)^{\beta-1}|F(x, t, y, s)| d s d t \\
& +\frac{1}{\Gamma(\alpha) \Gamma(\beta)} \int_{a}^{x} \int_{y}^{d}(t-a)^{\alpha-1}(d-s)^{\beta-1}|F(x, t, y, s)| d s d t \\
& +\frac{1}{\Gamma(\alpha) \Gamma(\beta)} \int_{a}^{x} \int_{c}^{y}(t-a)^{\alpha-1}(s-c)^{\beta-1}|F(x, t, y, s)| d s d t .
\end{aligned}
$$

for any $(x, y) \in \Lambda$.

Inasmuch as $f: \Lambda \rightarrow \mathbb{R}$ is of bounded variation on $[x, b] \times[y, d]$, one possess

$$
\begin{aligned}
|F(x, t, y, s)| & =|f(t, s)-f(t, y)-f(x, s)+f(x, y)| \\
& \leq \bigvee_{t}^{x} \bigvee_{s}^{y}(f) \leq \bigvee_{x} \bigvee_{y}^{d}(f) .
\end{aligned}
$$

For the first integral in the right hand side of the statement (4.5), we deduce that

$$
\begin{aligned}
& \frac{1}{\Gamma(\alpha) \Gamma(\beta)} \int_{x}^{b} \int_{y}^{d}(b-t)^{\alpha-1}(d-s)^{\beta-1}|F(x, t, y, s)| d s d t \\
& \leq \frac{(b-x)^{\alpha}(d-y)^{\beta}}{\Gamma(\alpha+1) \Gamma(\beta+1)} \bigvee_{x}^{b} \bigvee_{y}^{d}(f) .
\end{aligned}
$$

In a similar way, taking into account the other three integrals in the right hand side of (4.5) by considering that $f: \Lambda \rightarrow \mathbb{R}$ is of bounded variation on $[a, x] \times[c, y],[a, x] \times$ 
$[y, d]$ and $[x, b] \times[c, y]$, we reach the desired first inequality in (4.4). The second inequality in (4.4) is proved above.

Corollary 5. With the assumption of the Theorem 3, we have the midpoint type inequality

$$
\begin{aligned}
& \mid J_{\frac{a+b}{2}+\frac{c+d}{2}+}^{\alpha, \beta} f(b, d)+J_{\frac{a+b}{2}+, \frac{c+d}{2}-}^{\alpha, \beta} f(b, c)+J_{\frac{a+b}{2}-\frac{c+d}{2}+}^{\alpha, \beta} f(a, d)+J_{\frac{a+b}{2}-, \frac{c+d}{2}-}^{\alpha, \beta} f(a, c) \\
& \quad-\frac{(d-c)^{\beta}}{2^{\beta-1} \Gamma(\beta+1)}\left[J_{\frac{a+b}{2}+}^{\alpha} f\left(b, \frac{c+d}{2}\right)+J_{\frac{a+b}{2}-}^{\alpha} f\left(a, \frac{c+d}{2}\right)\right] \\
& \quad-\frac{(b-a)^{\alpha}}{2^{\alpha-1} \Gamma(\alpha+1)}\left[J_{\frac{c+d}{2}+,}^{\beta} f\left(\frac{a+b}{2}, d\right)+J_{\frac{c+d}{2}-,}^{\beta} f\left(\frac{a+b}{2}, c\right)\right] \\
& \quad+\frac{(b-a)^{\alpha}(d-c)^{\beta}}{2^{\alpha+\beta-2} \Gamma(\alpha+1) \Gamma(\beta+1)} f\left(\frac{a+b}{2}, \frac{c+d}{2}\right) \mid \\
& \leq \frac{(b-a)^{\alpha}(c-d)^{\beta}}{2^{\alpha+\beta} \Gamma(\alpha+1) \Gamma(\beta+1)} \bigvee_{a}^{d} \bigvee_{c}^{d}(f) .
\end{aligned}
$$

Theorem 4. Let $f: \Lambda \rightarrow \mathbb{R}$ be a function of bounded variation on $\Lambda$ in $\mathbb{R}^{2}$. Then, for any $(x, y) \in \Lambda$, we have

$$
\begin{aligned}
& \mid \frac{J_{b-, d-}^{\alpha, \beta} f(a, c)+J_{b-, c+}^{\alpha, \beta} f(a, d)+J_{a+, d-}^{\alpha, \beta} f(b, c)+J_{a+, c+-}^{\alpha, \beta} f(b, d)}{4} \\
& \quad-\frac{1}{2} \frac{(d-c)^{\beta}}{\Gamma(\beta+1)}\left[J_{b-}^{\alpha} f(a, y)+J_{a+}^{\alpha} f(b, y)\right] \\
& \quad-\frac{1}{2} \frac{(b-a)^{\alpha}}{\Gamma(\alpha+1)}\left[J_{d-,}^{\beta} f(x, c)+J_{c+,}^{\beta} f(x, d)\right]+\frac{(b-a)^{\alpha}(d-c)^{\beta}}{\Gamma(\alpha+1) \Gamma(\beta+1)} f(x, y) \mid \\
& \leq \frac{1}{4}\left\{A(x) C(y) \bigvee_{a}^{x} \bigvee_{c}^{y}(f)+A(x) D(y) \bigvee_{a}^{x} \bigvee_{y}^{d}(f)\right. \\
& \left.\quad+B(x) C(y) \bigvee_{x}^{b} \bigvee_{c}^{y}(f)+B(x) D(y) \bigvee_{x}^{b} \bigvee_{y}^{d}(f)\right\} \\
& \leq \frac{1}{4 \Gamma(\alpha+1) \Gamma(\beta+1)}\left[(b-a)^{\alpha}+\left|(x-a)^{\alpha}-(b-x)^{\alpha}\right|\right] \\
& \quad \times\left[(d-c)^{\beta}+\left|(y-c)^{\beta}-(d-y)^{\beta}\right|\right] \bigvee_{a}^{b} \bigvee_{c}^{d}(f)
\end{aligned}
$$

where

$$
A(x)=\frac{(b-a)^{\alpha}-(b-x)^{\alpha}+(x-a)^{\alpha}}{\Gamma(\alpha+1)},
$$




$$
\begin{aligned}
& B(x)=\frac{(b-a)^{\alpha}-(x-a)^{\alpha}+(b-x)^{\alpha}}{\Gamma(\alpha+1)}, \\
& C(y)=\frac{(d-c)^{\beta}-(d-y)^{\beta}+(y-c)^{\beta}}{\Gamma(\beta+1)}, \\
& D(y)=\frac{(d-c)^{\beta}-(y-c)^{\beta}+(d-y)^{\beta}}{\Gamma(\beta+1)} .
\end{aligned}
$$

Proof. Should we take absolute value of (3.6), from the modulus property of the integral, we get the inequality

$$
\begin{aligned}
& \mid \frac{J_{b-, d-}^{\alpha, \beta} f(a, c)+J_{b-, c+}^{\alpha, \beta} f(a, d)+J_{a+, d-}^{\alpha, \beta} f(b, c)+J_{a+, c+-}^{\alpha, \beta} f(b, d)}{4} \\
& \quad-\frac{1}{2} \frac{(d-c)^{\beta}}{\Gamma(\beta+1)}\left[J_{b-}^{\alpha} f(a, y)+J_{a+}^{\alpha} f(b, y)\right] \\
& \quad-\frac{1}{2} \frac{(b-a)^{\alpha}}{\Gamma(\alpha+1)}\left[J_{d-,}^{\beta} f(x, c)+J_{c+,}^{\beta} f(x, d)\right]+\frac{(b-a)^{\alpha}(d-c)^{\beta}}{\Gamma(\alpha+1) \Gamma(\beta+1)} f(x, y) \mid \\
& \leq \frac{1}{4} \frac{1}{\Gamma(\alpha) \Gamma(\beta)}\left\{\int _ { a } ^ { b } \int _ { c } ^ { d } \left[(t-a)^{\alpha-1}(s-c)^{\beta-1}+(t-a)^{\alpha-1}(d-s)^{\beta-1}\right.\right. \\
& \left.\left.\quad+(b-t)^{\alpha-1}(s-c)^{\beta-1}+(b-t)^{\alpha-1}(d-s)^{\beta-1}\right]|F(x, t, y, s)| d s d t\right\} .
\end{aligned}
$$

Considering that $f: \Lambda \rightarrow \mathbb{R}$ is of bounded variation on $\Lambda$ when calculating integral of the first of the four expressions given in the summation form in the right-hand side of the above inequality, it is easy to see that

$$
\begin{aligned}
& \frac{1}{4} \frac{1}{\Gamma(\alpha) \Gamma(\beta)} \int_{a}^{b} \int_{c}^{d}(t-a)^{\alpha-1}(s-c)^{\beta-1}|F(x, t, y, s)| d s d t \\
& \leq \frac{1}{4} \frac{1}{\Gamma(\alpha) \Gamma(\beta)}\left\{\bigvee_{a}^{x} \bigvee_{c}^{y}(f) \int_{a}^{x} \int_{c}^{y}(t-a)^{\alpha-1}(s-c)^{\beta-1} d s d t\right. \\
& +\bigvee_{a}^{x} \bigvee_{y}^{d}(f) \int_{a}^{x} \int_{y}^{d}(t-a)^{\alpha-1}(s-c)^{\beta-1} d s d t \\
& +\bigvee_{x}^{b} \bigvee_{c}^{y}(f) \int_{x}^{b} \int_{c}^{y}(t-a)^{\alpha-1}(s-c)^{\beta-1} d s d t
\end{aligned}
$$




$$
\left.+\bigvee_{x}^{b} \bigvee_{y}^{d}(f) \int_{x}^{b} \int_{y}^{d}(t-a)^{\alpha-1}(s-c)^{\beta-1} d s d t\right\}
$$

If the above integrals are calculated, one has the inequality

$$
\begin{aligned}
\frac{1}{4} & \frac{1}{\Gamma(\alpha) \Gamma(\beta)} \int_{a}^{b} \int_{c}^{d}(t-a)^{\alpha-1}(s-c)^{\beta-1}|F(x, t, y, s)| d s d t \\
\leq & \frac{1}{4} \frac{(x-a)^{\alpha}}{\Gamma(\alpha+1)} \frac{(y-c)^{\beta}}{\Gamma(\beta+1)} \bigvee_{a}^{x} \bigvee_{c}^{y}(f)+\frac{1}{4} \frac{(x-a)^{\alpha}}{\Gamma(\alpha+1)} \frac{(d-c)^{\beta}-(y-c)^{\beta}}{\Gamma(\beta+1)} \bigvee_{a}^{x} \bigvee_{y}^{d}(f) \\
& +\frac{1}{4} \frac{(b-a)^{\alpha}-(x-a)^{\alpha}}{\Gamma(\alpha+1)} \frac{(y-c)^{\beta}}{\Gamma(\beta+1)} \bigvee_{x}^{b} \bigvee_{c}^{y}(f) \\
& +\frac{1}{4} \frac{(b-a)^{\alpha}-(x-a)^{\alpha}}{\Gamma(\alpha+1)} \frac{(d-c)^{\beta}-(y-c)^{\beta}}{\Gamma(\beta+1)} \bigvee_{x}^{b} \bigvee_{y}^{d}(f) .
\end{aligned}
$$

If we sum all the resulting inequalities side by side after having been similarly calculated integrals of the other three expressions in (4.6), the first inequality can be readily obtained.

The second inequality can be easilly proved by using the facts (4.3). The proof is thus completed.

Corollary 6. Suppose that all the assumptions of Theorem 4 hold. If we choose $x=\frac{a+b}{2}$ and $y=\frac{c+d}{2}$, then we have

$$
\begin{aligned}
& \mid \frac{J_{b-, d-}^{\alpha, \beta} f(a, c)+J_{b-, c+}^{\alpha, \beta} f(a, d)+J_{a+, d-}^{\alpha, \beta} f(b, c)+J_{a+, c+-}^{\alpha, \beta} f(b, d)}{4} \\
& \quad-\frac{1}{2} \frac{(d-c)^{\beta}}{\Gamma(\beta+1)}\left[J_{b-}^{\alpha} f\left(a, \frac{c+d}{2}\right)+J_{a+}^{\alpha} f\left(b, \frac{c+d}{2}\right)\right] \\
& \quad-\frac{1}{2} \frac{(b-a)^{\alpha}}{\Gamma(\alpha+1)}\left[J_{d-,}^{\beta} f\left(\frac{a+b}{2}, c\right)+J_{c+,}^{\beta} f\left(\frac{a+b}{2}, d\right)\right] \\
& \quad+\frac{(b-a)^{\alpha}(d-c)^{\beta}}{\Gamma(\alpha+1) \Gamma(\beta+1)} f\left(\frac{a+b}{2}, \frac{c+d}{2}\right) \mid \\
& \leq \frac{1}{4} \frac{(b-a)^{\alpha}(c-d)^{\beta}}{\Gamma(\alpha+1) \Gamma(\beta+1)} \bigvee_{a}^{b} \bigvee_{c}^{d}(f) .
\end{aligned}
$$




\section{REFERENCES}

[1] G. Anastassiou, M. Hooshmandasl, A. Ghasemi, and F. Moftakharzadeh, "Montgomery identities for fractional integrals and related fractional inequalities," J. Inequal. Pure Appl. Math, vol. 10, no. 4, pp. 1-6, 2009.

[2] H. Budak and M. Z. Sarikaya, "A new generalization of ostrowski type inequality for mappings of bounded variation," Lobachevskii Journal of Mathematics, 2015.

[3] H. Budak and M. Z. Sarikaya, "A companion of generalization of ostrowski type inequalities for functions of two variables with bounded variation," Applied and Computational Mathematics, vol. 15 , no. 3, pp. 297-312, 2016.

[4] H. Budak and M. Z. Sarikaya, "On generalization ostrowski type inequalities for functions of two variables with bounded variation and applications," Palestine Journal of Mathematics, vol. 5, no. 1, pp. 86-97, 2016.

[5] H. Budak and M. Z. Sarikaya, "On ostrowski type inequalities for functions of two variables with bounded variation," International Journal of Analysis and Applications, vol. 12, no. 2, pp. 142156, 2016.

[6] H. Budak and M. Z. Sarikaya, "A companion of ostrowski type inequalities for mappings of bounded variation and some applications," Transactions of A. Razmadze Mathematical Institute, vol. 171, no. 2, pp. 136-143, 2017.

[7] H. Budak, M. Z. Sarıkaya, and A. Qayyum, "Improvement in companion of ostrowski type inequalities for mappings whose first derivatives are of bounded variation and applications," Filomat, vol. 31, no. 16, 2017.

[8] P. Cerone, S. S. Dragomir, and C. E. Pearce, "A generalized trapezoid inequality for functions of bounded variation," Turkish Journal of Mathematics, vol. 24, no. 2, pp. 147-163, 2000.

[9] J. A. Clarkson and C. R. Adams, "On definitions of bounded variation for functions of two variables," Transactions of the American Mathematical Society, vol. 35, no. 4, pp. 824-854, 1933.

[10] Z. Dahmani et al., "On minkowski and hermite-hadamard integral inequalities via fractional integration," Annals of Functional Analysis, vol. 1, no. 1, pp. 51-58, 2010.

[11] S. S. Dragomir, "The ostrowski integral inequality for mappings of bounded variation," Bulletin of the Australian Mathematical Society, vol. 60, no. 3, pp. 495-508, 1999.

[12] S. S. Dragomir and C. Pearce, "Selected topics on hermite-hadamard inequalities and applications," Mathematics Preprint Archive, vol. 2003, no. 3, pp. 463-817, 2003.

[13] S. S. Dragomir, "On the midpoint quadrature formula for mappings with bounded variation and applications," Kragujevac J. Math, vol. 22, no. 1, pp. 13-19, 2000.

[14] S. S. Dragomir, "Ostrowski type inequalities for riemann\% liouville fractional integrals of bounded variation, hölder and lipschitzian functions," Preprint RGMIA Res. Rep. Coll, vol. 20, 2017.

[15] S. S. Dragomir, "Composite ostrowski and trapezoid type inequalities for riemann liouville fractional integrals of functions with bounded variation," in Advanced Topics in Mathematical Analysis. CRC Press, 2019, pp. 425-448.

[16] S. Dragomir, "On the ostrowski's integral inequality for mappings with bounded variation and applications," Mathematical Inequalities and Applications, vol. 4, pp. 59-66, 2001.

[17] S. Erden, "Some perturbed inequalities of ostrowski type for funtions whose nth derivatives are of bounded," Iranian Journal of Mathematical Sciences and Informatics, in press, 2019.

[18] R. Gorenflo and F. Mainardi, Fractional calculus: integral and differential equations of fractional order. arXiv preprint arXiv:0805.3823, 2008.

[19] M. Jleli and B. Samet, "On hermite-hadamard type inequalities via fractional integrals of a function with respect to another function," J. Nonlinear Sci. Appl, vol. 9, no. 3, pp. 1252-1260, 2016. 
[20] M. A. Latif, S. Dragomir, and A. Matouk, "New inequalities of ostrowski type for co-ordinated convex functions via fractional integrals," J. Fract. Calc. Appl, vol. 2, no. 1, 2012.

[21] K. S. Miller and B. Ross, An introduction to the fractional calculus and fractional differential equations. Wiley, 1993.

[22] A. Ostrowski, "Über die absolutabweichung einer differentiierbaren funktion von ihrem integralmittelwert." Commentarii Mathematici Helvetici, vol. 10, pp. 226-227, 1937.

[23] J. E. Peajcariaac and Y. L. Tong, Convex functions, partial orderings, and statistical applications. Academic Press, 1992.

[24] I. Podlubny, Fractional differential equations: an introduction to fractional derivatives, fractional differential equations, to methods of their solution and some of their applications. Elsevier, 1998.

[25] M. Z. Sarıkaya, "On the hermite-hadamard-type inequalities for co-ordinated convex function via fractional integrals," Integral Transforms and Special Functions, vol. 25, no. 2, pp. 134-147, 2014.

[26] M. Z. Sarikaya and H. Budak, "Some hermite-hadamard type integral inequalities for twice differentiable mappings via fractional integrals," Facta Universitatis, Series: Mathematics and Informatics, vol. 29, no. 4, pp. 371-384, 2015.

[27] M. Z. Sarikaya and H. Budak, "Generalized hermite-hadamard type integral inequalities for fractional integrals," Filomat, vol. 30, no. 5, pp. 1315-1326, 2016.

[28] M. Z. Sarikaya and S. Erden, "On the weighted integral inequalities for convex function," Acta Universitatis Sapientiae Mathematica, vol. 6, no. 2, pp. 194-208, 2014.

[29] M. Z. Sarikaya, H. Filiz, and M. E. Kiris, "On some generalized integral inequalities for riemannliouville fractional integrals," Filomat, vol. 29, no. 6, pp. 1307-1314, 2015.

[30] M. Z. Sarikaya, E. Set, H. Yaldiz, and N. Başak, "Hermite-hadamard's inequalities for fractional integrals and related fractional inequalities," Mathematical and Computer Modelling, vol. 57, no. 9-10, pp. 2403-2407, 2013.

[31] Y. Zhang and J. Wang, "On some new hermite-hadamard inequalities involving riemann-liouville fractional integrals," Journal of Inequalities and Applications, vol. 2013, no. 1, p. 220, 2013.

Authors' addresses

\section{S. Erden}

Bartın University, Faculty of Science, Department of Mathematics, Bartın, Turkey.

E-mail address: erdensmt@gmail.com

\section{H. Budak}

Department of Mathematics, Faculty of Science and Arts, Düzce University, Düzce-Turkey

E-mail address: hsyn.budak@gmail.com

\section{Z. Sarkkaya}

Department of Mathematics, Faculty of Science and Arts, Düzce University, Düzce-Turkey

E-mail address: sarikayamz@gmail.com 